\title{
Basic image-processing operations by use of acousto-optics
}

\author{
Partha P. Banerjee, Dongqing Cao, and Ting-Chung Poon
}

\begin{abstract}
We describe some basic optical image-processing operations with acousto-optic (AO) Bragg diffraction. Instead of using frequency-plane filters, we place an AO cell behind the object. We then realize experimentally one-dimensional edge enhancement, which utilizes a high-pass filtering effect in the undiffracted order from the AO cell. A numerical simulation compares well with the experimental results. With two AO cells oriented orthogonally to each other, a second-order mixed derivative operation, evident from the four-corner enhancement of a square, is also demonstrated. (C) 1997 Optical Society of America
\end{abstract}

\section{Introduction}

An acousto-optic $(\mathrm{AO})$ cell is a device that produces a single (Bragg regime) diffracted light beam or multiple (Raman-Nath regime) diffracted light beams through the interaction of light and sound in the cell. AO devices have been used widely as optical signalprocessing components during the past three decades. The use of an AO cell as an image correlator in an optical heterodyning architecture was demonstrated independently by Whitman et al. ${ }^{1}$ and King et $a l .^{2}$ Two crossed Fourier plane Bragg cells have also been used by Prather and Mait ${ }^{3}$ and Mait et al. ${ }^{4}$ to implement two-dimensional (2-D) dynamic pointspread functions. AO cells were used in the Fourier plane as a dynamic spatial filter, resulting in morphological erosion and dilation operations. More recently Athale et al..$^{5}$ reported the implementation of dynamic arbitrary bipolar point-spread function image correlation and digital image subtraction with incoherent illumination. An AO cell was also used by Balakshy et al. ${ }^{6}$ to broadcast images into zeroth and first diffracted orders.

AO Bragg diffraction of profiled light beams can be explained ${ }^{7-10}$ with transfer functions in the spatialfrequency domain. Although the transfer functions

P. P. Banerjee and D. Cao are with the Department of Electrical and Computer Engineering and Center for Applied Optics, University of Alabama in Huntsville, Huntsville, Alabama 35899. T.-C. Poon is with the Optical Image Processing Laboratory, Bradley Department of Electrical Engineering, Virginia Polytechnic Institute and State University, Blacksburg, Virginia 24061.

Received 31 May 1996; revised manuscript received 3 December 1996.

0003-6935/97/143086-04\$10.00/0

(C) 1997 Optical Society of America of different orders are not the same, they do depend on common parameters such as the peak phase delay of the light through the sound cell $(\alpha)$ and the KleinCook parameter $(Q){ }^{11}$ Instead of using a frequencyplane filter for optical spatial filtering, one can use an AO cell placed behind the object. The filter characteristics are programmable because the transfer function can be changed by adjustment of the sound frequency, the sound amplitude, and the angle of incident light. For example, image differentiation may be realized because a change in the incident angle corresponds to a shift in the slope of the transfer function. ${ }^{12}$

In this paper we present both theoretical and experimental examples to demonstrate how images can be broadcast as well as processed with a novel $\mathrm{AO}$ spatial-filtering scheme. AO cells are not placed in the frequency plane. The fact that the position of the first-diffracted-order image can be changed by variation of the sound frequency allows the AO cell to broadcast the image electronically. Also, under certain conditions (as discussed in Section 2), the image of the zeroth or undiffracted order exhibits edge enhancement (high-pass filtering), while the broadcast image in the first diffracted order shows a low-pass filtered version of the input image. Theoretical predictions are based on numerical simulations with the discrete Fourier transform. Calculation results explain the edge-enhancement effect in the undiffracted-order image and the low-pass filtering effect in the first-diffracted-order image. We also present an extension to 2-D image processing with two one-dimensional (1-D) AO cells orthogonally oriented, and we demonstrate a second-order derivative operation leading to a four-corner enhancement of a square object. 


\section{Spatial Transfer Function of Bragg Regime AO Interaction}

In the Bragg regime the spatial transfer functions for undiffracted and first diffracted orders are given $a^{8,9}$

$$
\begin{aligned}
H_{0}\left(k_{x}\right)= & \exp \left[j\left(\frac{k_{x}^{2} L}{2 k_{0}}-\frac{k_{x} Q \Lambda_{0}}{4 \pi}\right)\right]\left\{\cos \left[\left(\frac{k_{x} Q \Lambda_{0}}{4 \pi}\right)^{2}+\left(\frac{\alpha}{2}\right)^{2}\right]^{1 / 2}\right. \\
& \left.+\left(\frac{j k_{x} Q \Lambda_{0}}{4 \pi}\right) \frac{\sin \left[\left(k_{x} Q \Lambda_{0} / 4 \pi\right)^{2}+(\alpha / 2)^{2}\right]^{1 / 2}}{\left[\left(k_{x} Q \Lambda_{0} / 4 \pi\right)^{2}+(\alpha / 2)^{2}\right]^{1 / 2}}\right\} \\
H_{1}\left(k_{x}\right)= & \exp \left[j\left(\frac{k_{x}^{2} L}{2 k_{0}}-\frac{k_{x} Q \Lambda_{0}}{4 \pi}\right)\right] \\
& \times\left(-j \frac{\alpha}{2}\right) \frac{\sin \left[\left(k_{x} Q \Lambda_{0} / 4 \pi\right)^{2}+(\alpha / 2)^{2}\right]^{1 / 2}}{\left[\left(k_{x} Q \Lambda_{0} / 4 \pi\right)^{2}+(\alpha / 2)^{2}\right]^{1 / 2}}
\end{aligned}
$$

respectively, where $Q$ is the Klein-Cook parameter, $\alpha$ represents the peak phase delay and is proportional to the sound pressure, $L$ is the interaction length, $\Lambda_{0}$ is the sound wavelength, $k_{0}$ denotes the propagation constant of light, and $k_{x}$ denotes the spatial frequency of diffracted light along the transverse $(x)$ direction. The propagational diffraction effect through the length of the AO cell is represented by the $\exp \left[j\left(k_{x}{ }^{2} L / 2 k_{0}\right)\right]$ phase term. The second part of the exponential in Eqs. (1) (the linear term in $k_{x}$ ) gives rise to a shift in the position of the pattern in the far field. 8,9 The remaining terms in Eqs. (1) characterize the AO interaction process. Equations (1) therefore show that, in essence, the propagational diffraction effects are decoupled from the $A O$ interaction process. In the derivation of Eqs. (1), sound-light interaction is confined to the $x-z$ plane with the light incident predominantly along the $z$ axis. Physically, $H_{0}$ and $H_{1}$ contain terms that constitute the complex amplitudes of the zeroth-order and the first-diffracted-order planewave components as a function of the angle (proportional to $k_{x}$ ).

For a fixed $Q$, the peak phase delay $\alpha$ can change the nature of the two transfer functions. ${ }^{8-10}$ In the current experiment, values of $\alpha$ are restricted to be less than $\pi$. Numerical calculation results show that, if $\alpha<\pi / 2$, the high-pass filtering characteristic of the zeroth-order transfer function and the low-pass filtering property of the first-order transfer function are not significant; but if $\pi / 2<\alpha<\pi$, the filtering is strong enough to process both undiffracted and diffracted images. Figure 1 shows the two transfer functions at $\alpha=0.3 \pi$ and $\alpha=0.6 \pi$. It is clear that, at $\alpha=0.6 \pi$, the zeroth-order transfer function exhibits strong high-pass filtering while the first-order transfer function demonstrates low-pass filtering. McNeill and Poon ${ }^{10}$ provide a detailed discussion of the dependence of the transfer functions on the peak phase delay.

\section{One-Dimensional Edge Enhancement}

The 1-D image-processing experiment was set up as shown in Fig. 2. Lenses 1 and 2 were used to
$|\mathbf{H}|$

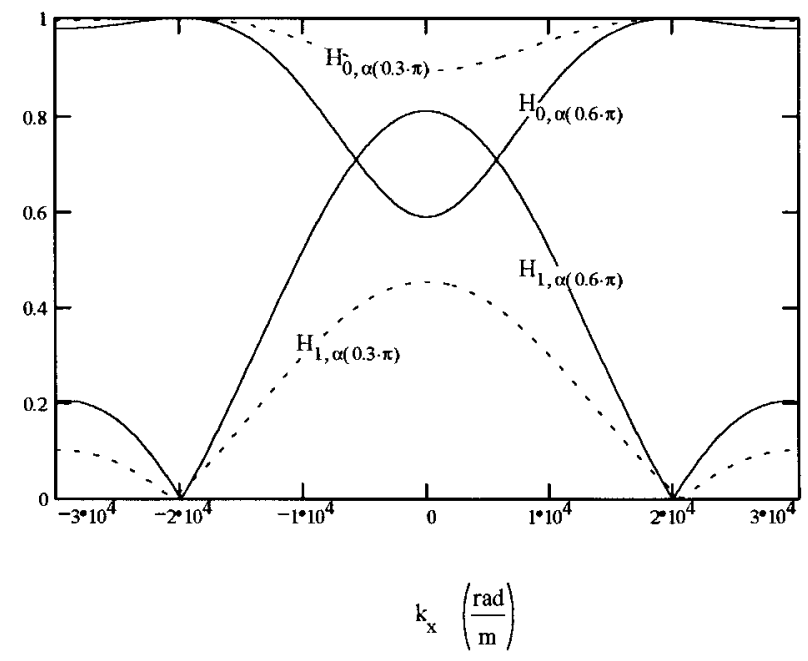

Fig. 1. Transfer functions for various values of $\alpha$ and $Q=27: H_{0}$ $(\alpha=0.3 \pi)$, zeroth-order transfer function for $\alpha=0.3 \pi$ (dotted curve); $H_{0}(\alpha=0.6 \pi)$, zeroth-order transfer function for $\alpha=0.6 \pi$ (solid curve); $H_{1}(\alpha=0.3 \pi)$, first-order transfer function for $\alpha=$ $0.3 \pi$ (dotted curve); and $H_{1}(\alpha=0.6 \pi)$, first-order transfer function for $\alpha=0.6 \pi$ (solid curve). The value of $Q=27$ is chosen to correspond to experimental conditions.

decrease the effective object size. The first AO cell was put immediately behind the object plane. The second AO cell was not used in this case. A He-Ne laser $(\lambda=633 \mathrm{~nm})$ was the light source. An IntraAction Corp. Model AOM-40 AO cell with a carrier frequency of $40 \mathrm{MHz}$ was used. The $\mathrm{AO}$ medium is flint glass with a refractive index of 1.68 , and the sound velocity in the material is approximately $3.96 \mathrm{~km} / \mathrm{s}$. The iris in the back focal plane of lens 3 was used to select different diffracted orders for display. Lenses 3 and 4 were used to image exactly the object on a CCD detector array of a Spiricon, Inc. laser beam analyzer. Therefore the propagational diffraction effect between the $\mathrm{AO}$ cell and the CCD camera (which, as explained above, includes diffraction through the AO cell but is decoupled from the interaction process) has been taken into account. The test object first used was a circle with transmission function $t(r, \theta)=\operatorname{circ}(r /$ $a$ ), where $a \approx 1 \mathrm{~mm}$.

On the basis of the above data, the light and sound frequencies, and the AO cell parameters, $Q$ and $\Lambda_{0}$ are found to be $Q \simeq 27$ and $\Lambda_{0} \simeq 0.07 \mathrm{~mm}$. The value

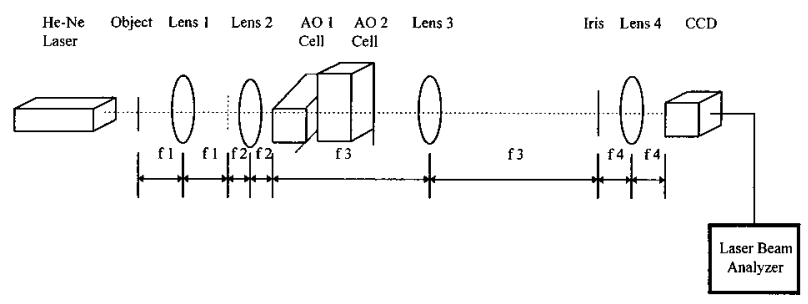

Fig. 2. Experimental setup. 


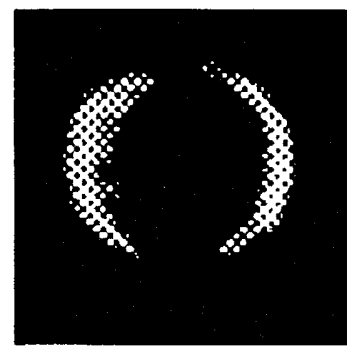

(a)

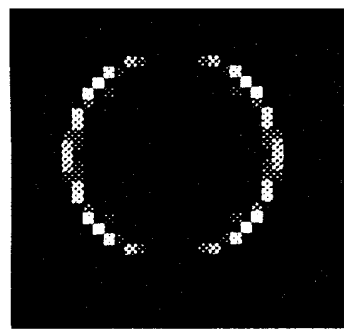

(c)

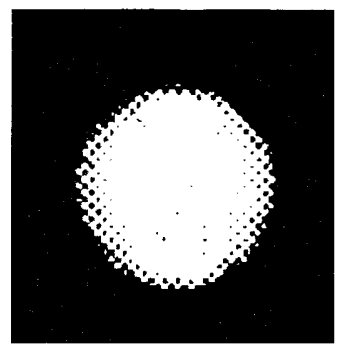

(b)

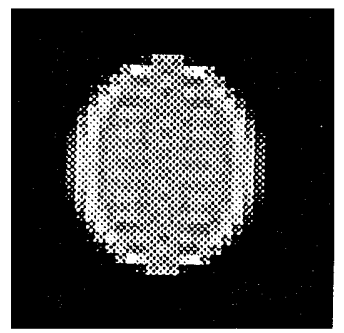

(d)
Fig. 3. Experimental and numerical simulation results for $Q=27$ and $\alpha=0.6 \pi$ with a circle pattern input object: (a) undiffracted image, experimental result; (b) diffracted image, experimental result; (c) zeroth-order intensity distribution from numerical simulations; (d) first-order intensity distribution from numerical simulations.

of $\alpha$ is calculated from the relation $11 \tan ^{2}(\alpha / 2)=I_{1} /$ $I_{0}$, where $I_{1}$ and $I_{0}$ are the first-order and zeroth-order optical intensities, respectively, that were measured experimentally without the test object.

Experimental results showing 1-D image processing of the circular aperture at $\alpha=0.6 \pi$ are given in Figs. 3(a) and 3(b). The zeroth-order image exhibits significant high-pass filtering with the near-vertical edges enhanced, and the first-order image demonstrates low-pass filtering with the edges smeared out. Because the AO cell interaction is confined in the $x-z$ plane, these effects are along the $x$ direction. Edge enhancement is less pronounced for small values of $\alpha$. A square transparency $t(x, y)=\operatorname{rect}(x / a) \operatorname{rect}(y / a)$ has been used as well. Results are similar to those shown in Figs. 3(a) and 3(b); vertical edges are enhanced and shown later in Section 5 [Fig. 4(b)].

\section{Numerical Simulations}

On the basis of the above parameters and the measured value of $\alpha$, a discrete Fourier transform method $^{13}$ was used to simulate the experiment. The transfer functions were multiplied with the angular plane-wave spectrum of the input object to derive the spectrum of the processed image at the exit of the $\mathrm{AO}$ cell. The inverse Fourier transform of the exit spectrum is equivalent in shape to the field pattern at the CCD detector (which was placed at the back focal plane of lens 4). The numerical results that correspond to the 1-D experiment described in Section 3 are shown in Figs. 3(c) and 3(d). The numerical simulations are in good agreement with the experimental results.

\section{Two-Dimensional Image Processing Experiment}

The extension of this technique to 2-D image processing requires that a second $\mathrm{AO}$ cell be added immediately behind the first $\mathrm{AO}$ cell shown in Fig. 2 and oriented orthogonally to the first one. The action of the two orthogonally oriented AO cells can be understood simply as the following: The amplitude $E_{1}$ of the undiffracted order of the first AO cell can be approximated with the transfer function [see Eq. (1a) and Fig. 1] as

$$
E_{1}(x, y)=\left(A_{1}+B_{1} \partial / \partial x\right) t(x, y)
$$

where $A_{1}$ is a function of the peak phase delay $\alpha$ and $B_{1}$ is a function of $\alpha$, the Klein-Cook parameter $Q$, and the sound wavelength $\Lambda_{0}$ in the first AO cell. It was noted experimentally that the second part of the right-hand side of Eq. (2) is dominant. After $E_{1}(x, y)$ passes through the second AO cell, the amplitude of the undiffracted order $E_{2}$ of the second cell can be

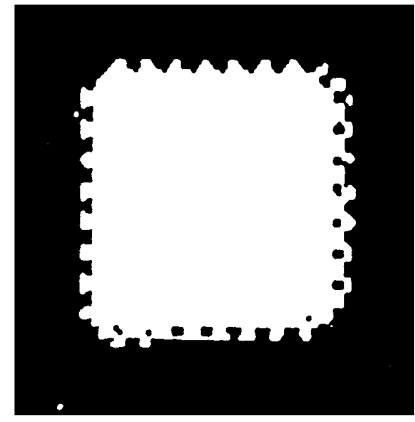

(a)

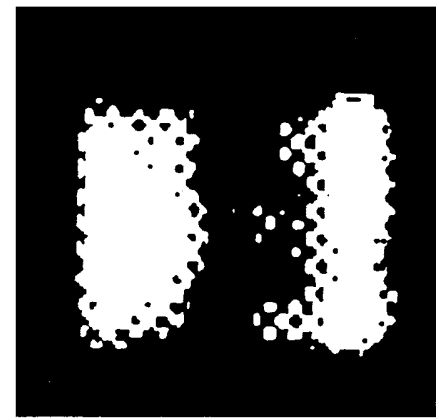

(b)

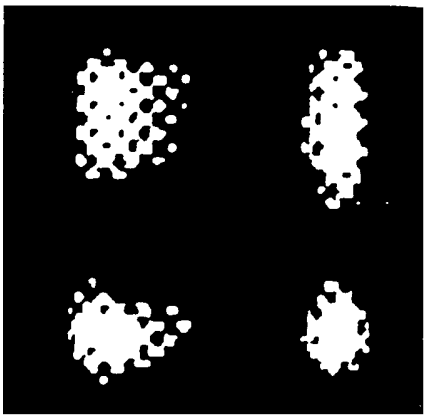

(c)

Fig. 4. Experimental four-corner enhancement results: (a) image without any AO interaction, (b) image with only 1-D AO interaction, and (c) image with two orthogonally oriented 1-D AO cells. 
expressed as

$$
\begin{aligned}
E_{2}(x, y) & =\left(A_{2}+B_{2} \partial / \partial y\right) E_{1}(x, y) \\
& \approx B_{1} B_{2} \partial^{2} / \partial y \partial x[t(x, y)],
\end{aligned}
$$

where $A_{2}$ and $B_{2}$ are functions of $\alpha$, and $Q$ and $\Lambda_{0}$ of the second $\mathrm{AO}$ cell are similar to $A_{1}$ and $B_{1}$.

A $2 \mathrm{~mm} \times 2 \mathrm{~mm}$ transparency (the size is demagnified by a factor of 2 with lenses 1 and 2) was used experimentally as the initial $t(x, y)$. The results, imaged upon and recorded by the CCD camera, are shown in Fig. 4. Figure 4(a) shows the original object; Fig. 4(b) shows vertical edge enhancement, as stated in Section 3, when only the first AO cell is active. With both $\mathrm{AO}$ cells in operation the second derivative operation is achieved and is evident by the four-corner enhancement of the square object, as shown in Fig. 4(c).

\section{Image Broadcasting}

As we stated in the introduction, the $\mathrm{AO}$ cell can also be used to broadcast images. The experimental setup as shown in Fig. 2 was used, but only the first AO cell was active, with a sound frequency of $\sim 40$ $\mathrm{MHz}$. It was observed that images can be broadcast into at least four diffracted orders along the $x$ direction with near Bragg angle incidence of the input light from the transparency. One can also change easily the spatial positions of the diffracted images by changing the frequency of the sound wave. It is conceivable that image broadcasting into a 2-D array can be effected by activation of both the $\mathrm{AO}$ cells, as shown in Fig. 2.

\section{Summary}

A novel programmable optical image-processing technique that makes use of AO cells is introduced. The filtering properties of $\mathrm{AO}$ cells can be used to realize image edge and corner enhancements. Both 1-D and 2-D image processing are possible, and numeri- cal simulations verify the $1-\mathrm{D}$ experimental results. The possibility of image broadcasting is also discussed. Rigorous theoretical analyses and numerical simulation of $2-\mathrm{D}$ image processing with $\mathrm{AO}$ cells are currently under way.

\section{References}

1. R. Whitman, A. Korpel, and S. Lotsoff, "Application of acoustic Bragg diffraction to optical processing techniques," in Proceedings of a Symposium on Modern Optics, J. Fox, ed. (Polytechnic, Brooklyn, N.Y. 1967), pp. 243-256.

2. M. King, W. R. Bennett, L. B. Lambert, and M. Arm, "Realtime electrooptical signal processors with coherent detection," Appl. Opt. 6, 1367-1375 (1967).

3. D. W. Prather and J. N. Mait, "Acousto-optic generation of two-dimensional spot arrays," Opt. Lett. 16, 1720-1722 (1991).

4. J. N. Mait, D. W. Prather, and R. A. Athale, "Acousto-optic processing with electronic feedback for morphological filtering," Appl. Opt. 31, 5688-5699 (1992).

5. R. A. Athale, J. Van Der Gracht, D. W. Prather, and J. W. Mait, "Incoherent optical image processing with acousto-optic pupilplane filtering," Appl. Opt. 34, 276-280 (1995).

6. V. I. Balakshy, "Scanning of Images," Sov. J. Quantum Electron. 6, 965-971 (1979).

7. A. Korpel, P. P. Banerjee, and C.-W. Tarn, "A unified treatment of spectral formalisms of light propagation and their application to acousto-optics," Opt. Commun. 97, 250-258 (1993).

8. P. P. Banerjee and C.-W. Tarn, "A Fourier transform approach to acoustooptic interactions in the presence of propagational diffraction," Acustica 74, 181-191 (1991).

9. M. R. Chatterjee, T.-C. Poon, and D. N. Sitter, Jr., "Transfer function formalism for strong acousto-optic Bragg diffraction of light beams with arbitrary profiles," Acustica 71, 81-92 (1990).

10. M. D. McNeill and T.-C. Poon, "Gaussian-beam profile shaping by acousto-optic Bragg diffraction,” Appl. Opt. 33, 4508-4515 (1994).

11. A. Korpel, Acousto-Optics (Marcel Dekker, New York, 1988), pp. 43-93.

12. V. I. Balakshy and A. G. Kukuskhn, "Visualization of phase objects in Bragg diffraction," Sov. Opt. Spectrosc. 64, 99-103 (1988).

13. J. W. Goodman, Introduction to Fourier Optics, 2nd ed. (McGraw-Hill, New York, 1996), pp. 4-27. 